\title{
Gravidezes Bem Sucedidas com Recurso a Fertilização In Vitro após Oclusão de Hidrossalpinge Unilateral com Essure ${ }^{\circledR}$
}

\author{
Successful Pregnancies Outcomes with the Use of In Vitro Fertilization \\ after Essure ${ }^{\circledR}$ Unilateral Hydrossalpinx Occlusion
}

Luís GUEDES-MARTINS 1,2,3, Joana MESQUITA-GUIMARÃES ${ }^{4}$, Márcia BARREIRO4

Acta Med Port 2014 May-Jun;27(3):342-348

RESUMO

Introdução: Desde há duas décadas que assistimos à publicação de vários estudos dedicados à avaliação da influência da hidrossalpinge nos resultados de técnicas de transferência de embriões. O objectivo deste trabalho é apresentar os resultados do tratamento e vigilância gestacional em mulheres com história de infertilidade - associada a hidrossalpinge unilateral, visível no exame ecográfico transvaginal - submetidas a oclusão unilateral com Essure ${ }^{\circledR}$ e tratamento subsequente com fertilização in vitro.

Material e Métodos: Análise prospectiva de uma amostra constituída por seis mulheres, com antecedentes de infertilidade e hidrossalpinge unilateral, entre Abril de 2010 e Maio de 2013. Em todos os casos procedemos a exclusão de hidrossalpinge recorrendo à colocação unilateral de um dispositivo Essure ${ }^{\circledR}$ por via histeroscópica antes da realização de um ciclo para fertilização in vitro.

Resultados: Das seis doentes submetidas a fertilização in vitro, quatro engravidaram (66,7\%). Destas, registaram-se duas gravidezes de termo sem intercorrências até ao parto e duas gravidezes encontram-se em vigilância, sem intercorrências conhecidas até à data. Das doentes que não engravidaram após fertilização in vitro $(n=2,33,3 \%)$, uma engravidou espontaneamente durante a vigilância subsequente.

Discussão: $O$ avanço da histeroscopia no tratamento de hidrossalpinge, recorrendo à implantação do dispositivo Essure ${ }^{\circledR}$, representa uma alternativa válida à abordagem laparoscópica.

Conclusão: Este estudo sugere que a oclusão tubar unilateral, induzida pelo dispositivo Essure ${ }^{\circledR}$, melhora os resultados do tratamento com fertilização in vitro em casos de infertilidade associada a hidrossalpinge unilateral, visível no exame ecográfico transvaginal.

Palavras-chave: Fertilização In Vitro; Histeroscopia; Infertilidade Feminina; Gravidez; Nascimento Vivo; Esterilização Tubária; Portugal.

\section{ABSTRACT}

Introduction: Since two decades we witnessed the publication of several studies devoted to the study of the influence of the presence of hydrosalpinx on the results of embryo transfer techniques. The aim of this study is to present the results of treatment and pregnancy outcomes in women with a history of infertility associated with unilateral hydrosalpinx, visible on vaginal ultrasound, which were subjected to unilateral occlusion with Essure ${ }^{\circledR}$ and subsequent treatment with in vitro fertilization.

Material and Methods: We performed a prospective analysis of a sample of 6 women, with a history of infertility and unilateral hydrosalpinx, between April 2010 to May 2013. In all cases we proceeded to unilateral hysteroscopic placement of the Essure ${ }^{\circledR}$ microinsert, prior to performing a cycle for in vitro fertilization.

Results: Of the 6 patients undergoing in vitro fertilization, 4 became pregnant (66.7\%). Of these, two were uneventful pregnancies until delivery and 2 are still under surveillance, without complications. Of the patients who did not become pregnant after in vitro fertilization ( $n=2,33.3 \%), 1$ conceived spontaneously during the subsequent monitoring.

Discussion: The advancement of hysteroscopy in the treatment of hydrosalpinx using the Essure ${ }^{\circledR}$ microinsert placement is a valid alternative to the laparoscopic approach.

Conclusion: This study suggests the effectiveness of unilateral tubal occlusion caused by Essure ${ }^{\circledR}$ microinsert in improving outcomes of in vitro fertilization treatment in cases of infertility associated with unilateral hydrosalpinx, visible in the vaginal ultrasound.

Keywords: Fertilization in Vitro; Hysteroscopy; Infertility, Female; Pregnancy; Live Birth; Sterilization, Tubal; Portugal.

\section{INTRODUÇÃO}

Hidrossalpinge é uma palavra de origem grega que significa trompa de Falópio preenchida com água ou fluido. ${ }^{1,2}$

A influência negativa da presença de hidrosalpinge(s) sobre as taxas de sucesso da fertilização in vitro (IVF-ET) é, reconhecidamente, um tema de forte debate porque representa um problema frequente e com abordagem, por vezes, complexa. ${ }^{3-11}$ A teoria aceite actualmente é que o fluido presente no lúmen tubar dilatado desempenha um papel causal na redução da taxa de gravidez resultante de técnicas de transferência de embriões. ${ }^{12-15}$ Acredita-se, por- tanto, que o liquido da(s) trompa(s) afectada(s) ocupa uma posição chave que resulta num prejuízo significativo para o processo de implantação. Com efeito, qualquer intervenção cirúrgica capaz de bloquear a comunicação entre a trompa e a cavidade uterina tem o potencial teórico de eliminar o vazamento do fluido da hidrossalpinge e melhorar as taxas de gravidez. ${ }^{13-19}$ Esta hipótese tem beneficiado de evidência crescente pela ocorrência de gravidezes espontâneas, em mulheres com hidrossalpinge, após oclusão tubar unilateral da trompa afectada. ${ }^{12}$

1. Departamento de Biologia Experimental. Faculdade de Medicina da Universidade do Porto. Porto. Portugal.

2. Instituto de Biologia Molecular e Celular. Porto. Portugal.

3. Departamento da Mulher e da Medicina Reprodutiva. Centro Hospitalar do Porto. Porto. Portugal.

4. Centro de Procriação Medicamente Assistida. Centro Hospitalar do Porto. Porto. Portugal.

Recebido: 14 de Setembro de 2013 - Aceite: 07 de Novembro de 2013 | Copyright @ Ordem dos Médicos 2014 
O Essure ${ }^{\circledR}$ é um dispositivo para esterilização definitiva, introduzido por via histeroscópica, apresenta $4 \mathrm{~cm}$ de comprimento e 1-2 mm de espessura. É composto por espirais de ácido inoxidável, tereftalato de polietileno e um revestimento de níquel-titânio exterior (nilitol). A utilização de Essure ${ }^{\circledR}$ é uma modalidade de tratamento relativamente nova para o efeito de oclusão tubar propriamente dita e com vantagens expectáveis em doentes com contraindicação para laparoscopia e/ou anestesia geral. ${ }^{20-25}$ Pode ser colocado em regime de ambulatório sem necessidade de anestesia e fora do contexto do bloco operatório.

A hidrossalpinge visível em ecografia transvaginal é uma entidade clínica que parece apresentar um pior prognóstico reprodutivo se não for ocluída ${ }^{1,2,7,9,14}$.

O objectivo deste trabalho é apresentar os resultados do tratamento e seguimento gestacional em mulheres com história de infertilidade - associada a hidrossalpinge unilateral, visível no exame ecográfico transvaginal - submetidas a oclusão unilateral com Essure ${ }^{\circledR}$ e tratamento subsequente com IVF-ET.

\section{MATERIAL E MÉTODOS}

Realizámos um estudo observacional prospectivo de uma série constituída por seis mulheres, com antecedentes de infertilidade e hidrossalpinge unilateral visível no exame ecográfico transvaginal, entre Abril de 2010 e Maio de 2013. Em todos os casos procedemos à exclusão de hidrossalpinge recorrendo à colocação unilateral de um dispositivo Essure ${ }^{\circledR}$ por via histeroscópica antes da realização de um ciclo para IVF-ET. Idade superior a 40 anos e a ausência de indicação para IVF-ET foram considerados critérios de exclusão para a participação no estudo. As doentes foram informadas acerca dos benefícios e riscos potenciais, relativos à oclusão tubar histeroscópica com Essure ${ }^{\circledR}$, de forma verbal e escrita. Todas as doentes assinaram um consentimento informado para inclusão na investigação proposta, publicação dos dados recolhidos e respectivos desfechos quer da técnica de procriação medicamente assistida quer dos resultados gestacionais. O protocolo do estudo foi aprovado no Serviço de Ginecologia do Centro Hospitalar do Porto e foi requerida a respetiva autorização institucional para publicação dos dados que constam no trabalho apresentado.

Em cada caso, procedemos à colheita de dados epidemiológicos como a idade, IMC (Índice de Massa Corporal), duração e tipo de infertilidade, confirmação ecográfica da existência de hidrossalpinge unilateral, a existência de tratamentos anteriores (e respectivos resultados) e a existência eventual de contraindicação para a realização de laparoscopia. Todos os procedimentos foram efectuados em
I

II
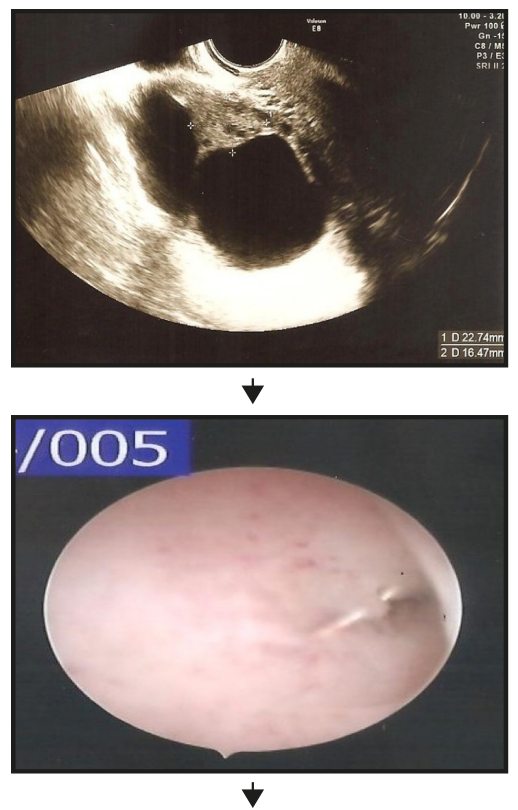

III

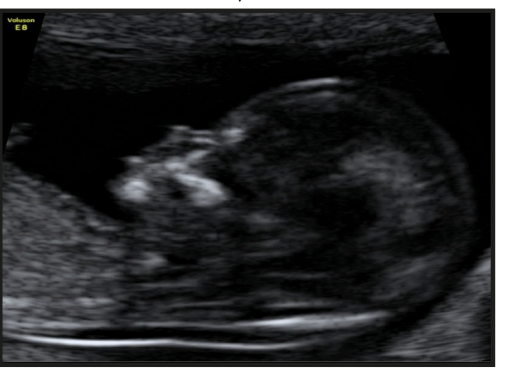

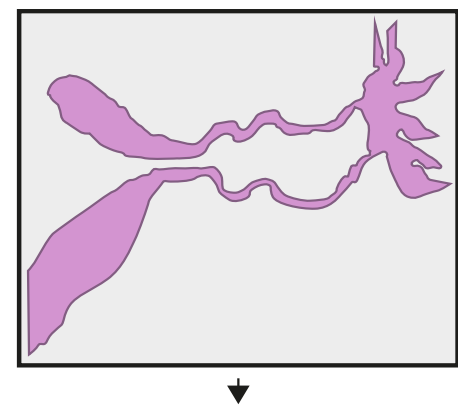
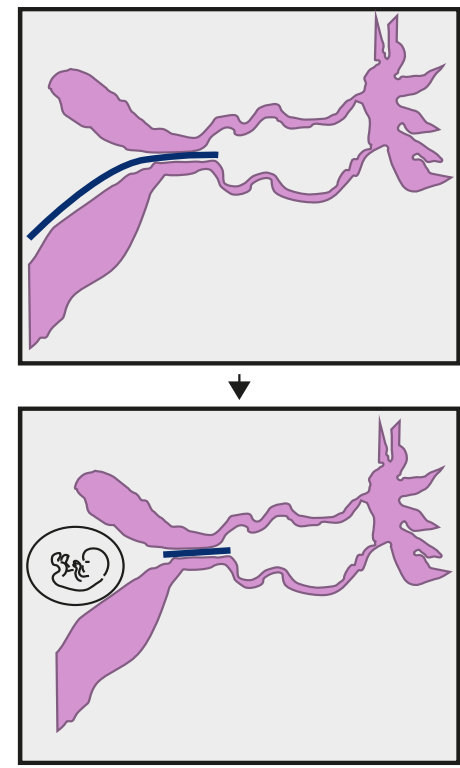

Figura 1 - Hidrossalpinge antes (I) e após (II-III) colocação de Essure ${ }^{\circledR}$. Gravidez às 12 semanas e 5 dias, bem sucedida, após ciclo de IVF-ET (III) 
regime de ambulatório e o critério de seleção para colocação de Essure ${ }^{\circledR}$ foi a presença de hidrossalpinge unilateral (independentemente da presença/ausência de indicação para laparoscopia).

A colocação de Essure ${ }^{\circledR}$ foi efectuada por via histeroscópica, sem recurso a anestesia, durante a segunda semana do ciclo menstrual. Todas as doentes foram medicadas com misoprostol $0.2 \mathrm{mg}$ (um comprimido de $0.2 \mathrm{mg}$ previamente humedecido e colocado por via vaginal) no dia anterior ao procedimento e todos os procedimentos foram efectuados sob cobertura antibiótica com Azitromicina 1g, por via oral, administrada meia hora antes da realização do mesmo. $\mathrm{O}$ material utilizado incluiu um histeroscópio Storz ${ }^{\circledR} / 5 \mathrm{~mm}$ com canal de trabalho e a histerodistensão foi conseguida com recurso a soro fisiológico. Após visualização dos óstios tubares procedemos à introdução de um dispositivo Essure ${ }^{\circledR}$, unilateralmente na trompa afectada, com o objectivo de deixar apenas uma ou duas espirais visíveis na cavidade uterina. Com efeito, registámos, para cada caso, o número de espirais visíveis na cavidade após libertação do dispositivo.

Cerca de três meses após, constatámos a localização esperada dos implantes recorrendo a radiografia pélvica e ecografia transvaginal bidimensional, complementada com ecografia tridimensional.

Posteriormente, as doentes foram submetidas a um ciclo de IVF-ET de acordo com o protocolo do serviço. Os desfechos da técnica e o seguimento de eventual gravidez foram registados (Fig.1). Procedemos, finalmente, à análise estatística descritiva dos dados obtidos.

\section{RESULTADOS}

A idade e o índice de massa corporal médios das doentes admitidas no estudo foi 3,.7 anos ( $\min .29$; máx. 36) e $24,3 \mathrm{Kg} / \mathrm{m}^{2}$ (min. 22,3; máx. 30,1), respectivamente. O tempo médio de infertilidade foi de 4,7 anos (min. 2; máx. 8). Das seis doentes inférteis contempladas pelo estudo, quatro apresentaram história de IVF-ET prévias (66,7\%) mal sucedidas. Apenas uma das doentes apresentou história de gravidez espontânea prévia: tratou-se de uma gravidez ectópica tubar, abordada cirurgicamente com salpingectomia laparotómica (um ano antes de ser admitida no Centro de Procriação Medicamente Assistida deste Hospital) - Tabela 1 . Relativamente às causas de infertilidade, em todas as doentes apresentadas, para além do factor tubar foi reconhecida a presença de factor masculino.

Em todos os casos $(n=6)$ foi constatada a existência de hidrossalpinge unilateral, diagnosticada por ecografia transvaginal, e todas as doentes aceitaram a oclusão tubar unilateral com Essure ${ }^{\circledR}$ embora, apenas uma doente apresentasse contraindicação para a realização de laparoscopia por diagnóstico de 'pelve congelada' em laparoscopia prévia (doente 3).

Os procedimentos foram efectuados pelo mesmo cirurgião, sem recurso a anestesia e em regime de ambulatório (conforme descrição prévia na secção 'Material e Métodos'). O número médio de espirais visualizadas na cavidade uterina, imediatamente após a colocação histeroscópica do implante, foi de 1,8 (min. 1; máx. 2) - Tabela 1. Todos os procedimentos decorreram sem complicações. Nenhuma doente necessitou de internamento e não foi identificada a ocorrência de patologia endocavitária concomitante.

Cerca de três meses após, constatámos a localização esperada dos implantes, de acordo com o protocolo do estudo. Nenhuma doente foi submetida a histerossalpingografia para confirmação da normal localização dos implantes. A informação fornecida pela radiografia pélvica e pela ecografia transvaginal bidimensional/tridimensional foi considerada esclarecedora em todos os casos.

O tempo médio calculado entre a data em que foi colocado o Essure ${ }^{\circledR}$ e a IVF-ET foi de sete meses (min. 5; máx. 8) e foram transferidos dois embriões por ciclo IVF-ET, de acordo com o protocolo em vigor do Centro de Procriação Medicamente Assistida onde decorreu a investigação (em nenhum caso foi oferecida a hipótese de elective single embryo transfer). Os respectivos desfechos são apresentados na Tabela 2.

Tabela 1 - Características gerais da amostra onde se procedeu a colocação de Essure ${ }^{\circledR}$

\begin{tabular}{|c|c|c|c|c|c|c|}
\hline Doente & $\begin{array}{l}\text { Idade } \\
\text { (anos) }\end{array}$ & $\begin{array}{c}\text { Duração da } \\
\text { infertilidade } \\
\text { (anos) }\end{array}$ & $\begin{array}{l}\text { FIV } \\
\text { prévia }\end{array}$ & $\begin{array}{c}\mathbf{N}^{\circ} \text { de gravidezes } \\
\text { prévias }\end{array}$ & $\begin{array}{l}\text { Hidrossalpinge } \\
\text { unilateral }\end{array}$ & $\begin{array}{l}N^{\circ} \text { de espirais } \\
\text { visíveis na } \\
\text { cavidade uterina }\end{array}$ \\
\hline 1 & 29 & 3 & 0 & $1^{*}$ & Sim & 2 \\
\hline 2 & 30 & 6 & 1 & 0 & Sim & 1 \\
\hline 3 & 31 & 5 & 0 & 0 & Sim & 2 \\
\hline 4 & 36 & 2 & 1 & 0 & Sim & 2 \\
\hline 5 & 34 & 4 & 1 & 0 & Sim & 2 \\
\hline 6 & 33 & 8 & 1 & 0 & Sim & 2 \\
\hline
\end{tabular}


Tabela 2 - Resultados de FIV-TE e vigilância da gravidez

\begin{tabular}{|c|c|c|c|c|c|}
\hline Doente & $\begin{array}{c}\text { Tempo Essure }{ }^{\circledR}-\text {-Ciclo } \\
\text { (meses) }\end{array}$ & $\begin{array}{l}N^{\circ} \text { embriões } \\
\text { transferidos }\end{array}$ & $\begin{array}{l}\text { № de embriões } \\
\text { implantados }\end{array}$ & $\begin{array}{c}\mathrm{N}^{\circ} \text { de } \\
\text { abortamentos }\end{array}$ & Gravidez \\
\hline 1 & 5 & 2 & 2 & 1 & $\begin{array}{l}\text { Sem intercorrências, parto } \\
\text { eutócico às } 39 \text { semanas } \\
\text { de recém-nascido do sexo } \\
\text { feminino }(p=2900 \mathrm{~g})\end{array}$ \\
\hline 2 & 7 & 2 & 1 & 0 & $\begin{array}{l}\text { Sem intercorrências, parto } \\
\text { distócico por cesariana } \\
\text { às } 39 \text { semanas de recém- } \\
\text { nascido do sexo masculino } \\
\qquad(p=3030 \mathrm{~g})\end{array}$ \\
\hline 3 & 7 & 2 & 0 & 0 & - \\
\hline 4 & 8 & 2 & 1 & 0 & $\begin{array}{l}\text { Gravidez simples, } 19 \\
\text { semanas, sem } \\
\text { intercorrências até à data }\end{array}$ \\
\hline 5 & 8 & 2 & 2 & 0 & $\begin{array}{c}\text { Gravidez gemelar } \\
\text { bicorionica/biamniotica, } \\
22 \text { semanas, sem } \\
\text { intercorrências até à data }\end{array}$ \\
\hline 6 & - & - & - & - & $\begin{array}{c}\text { Gravidez simples } \\
\text { espontânea, } 37 \text { semanas, } \\
\text { sem intercorrências até à } \\
\text { data }\end{array}$ \\
\hline
\end{tabular}

Das seis doentes submetidas a IVF-ET, quatro engravidaram $(66,7 \%)$. Destas, registámos duas gravidezes de termo sem intercorrências até ao parto e duas gravidezes encontram-se em vigilância, sem intercorrências conhecidas até à data. Das doentes que não engravidaram $(n,=, 2$, $33,3 \%$ ), uma engravidou espontaneamente (doente 6) durante a vigilância subsequente. A doente 3 aguarda realização de ciclo IVF-ET.

Considerando os aspectos relativos à vigilância das gravidezes em curso e os desfechos nas duas gravidezes de termo, nunca foi efectuada menção à posição dos implantes nas ecografias obstétricas realizadas, não foram encontradas anomalias da placentação (como consequência de uma possível irritação miometrial), complicações infecciosas (endometrite, corioamnionite, p.e.) ou ocorrência de rotura prematura de membranas.

\section{DISCUSSÃO}

A patologia tubar representa uma indicação importante para IVF-ET, em mulheres com história de infertilidade. ${ }^{1-8}$ No caso particular da hidrossalpinge, vários estudos têm sido dedicados à influência da sua presença nos resultados do tratamento de fertilização in vitro.

Embora não totalmente concordantes, os resultados das investigações despertam a atenção para os efeitos ne- gativos do líquido/fluido tubar. Em particular, nestas doentes, reconheceu-se que a baixa osmolaridade e as baixas concentrações de lactatos e de proteínas no líquido acumulado na hidrossalpinge são responsáveis por uma diminuição da motilidade espermática. ${ }^{9}$

A IVF-ET foi inicialmente desenvolvida para superar a doença tubar intratável ou ausência de trompas. ${ }^{10}$ Posteriormente, evoluiu para uma modalidade terapêutica em quase todas as formas de infertilidade. ${ }^{11}$ No subgrupo de doentes cujo factor de infertilidade reconhecido é o tubar, este não representa uma única entidade e a hidrossalpinge é descrita como a forma com pior prognóstico. ${ }^{11}$ Evidência crescente foi demonstrada na ocorrência de gravidez espontânea em mulheres com história de infertilidade associada a hidrossalpinge unilateral e trompa contralateral normal, recorrendo a técnicas de oclusão ou exérese da trompa afectada. ${ }^{12,13}$

Com efeito, os procedimentos cirúrgicos, para interrupção da comunicação entre a trompa afectada e a cavidade uterina, sofreram melhorias técnicas importantes. A salpingectomia ou a oclusão tubária por via laparoscópica são actualmente consideradas como os tratamento de eleição em doentes que vão ser submetidas a IVF-ET ${ }^{14,26,27}$ sobretudo aquelas com hidrossalpinges ecograficamente objectiváveis. ${ }^{15}$ Trata-se de um procedimento simples, pouco inva- 
sivo, associado a uma recuperação rápida. ${ }^{14}$ Embora alvo de alguma suspeita, em estudos recentes, a salpingectomia não se associou a perda de reserva/resposta ovári$\mathrm{ca}^{16-19}$ em estimulações subsequentes. Por este motivo, a laparoscopia é reconhecida por alguns como o tratamento de eleição da hidrossalpinge, com laqueação e exérese tubar, traduzindo benefícios claros nos resultados após IVF-ET. ${ }^{28,29}$ Contudo, não é imune a críticas e apresenta como principal limitação o facto de ser necessariamente efectuada sob anestesia e em ambiente de bloco operatório.

O avanço da histeroscopia no tratamento de hidrossalpinges, recorrendo à implantação do dispositivo Essure ${ }^{\circledR}$, representa uma alternativa aos problemas colocados pela abordagem laparoscópica. ${ }^{21-25}$ A ausência da necessidade de anestesia geral, a diminuição do risco operatório, o benefício económico ${ }^{30-32}$ (resultante, essencialmente, da ausência de necessidade de internamento e rápida convalescença), a possibilidade de tratamento no mesmo tempo operatório de eventuais lesões intracavitárias (p.e. pólipos endometriais) e a redução do risco de gravidez ectópica tubar proximal (cornual e no 'coto' tubar remanescente pós-salpingectomia), são considerados por alguns ${ }^{21-25}$ como os principais argumentos para o avanço desta técnica, inicialmente desenvolvida para realização de contracepção definitiva.

Críticas significativas têm sido colocadas ao procedimento descrito pelo facto de não se conhecerem os efeitos da presença de Essure ${ }^{\circledR}$ na(s) trompa(s) afectada(s) sobre o desenvolvimento embrionário e fetal subsequente. Estudos de biomateriais, como o realizado por Wever et al, ${ }^{33}$ revelaram que a liga Niquel-Titânio pode ser considerada em implantes por ser biologicamente segura pois revelou ausência de actividade citotóxica, alérgica e genotóxica, semelhante ao observado no material de referência controle composto por aço AISI 316 LVM. Nas cerca de 20 gravidezes bem sucedidas, descritas na literatura, ${ }^{21-25}$ resultantes de IVF-ET em mulheres inférteis submetidas a oclusão tubar com Essure ${ }^{\circledR}$, não foi descrito qualquer efeito negativo sobre o desenvolvimento embrio-fetal. Consideramos, no entanto, que estudos adicionais com séries mais alargadas serão necessários para esclarecer estas afirmações e, particularmente, reforçar a inocuidade sobre a placentação, risco infeccioso e de prematuridade.

O número de espirais visíveis na cavidade uterina, após colocação de Essure ${ }^{\circledR}$, tem sido apresentado nas melhores séries com um máximo de quatro. ${ }^{22-25} \mathrm{Na}$ verdade, não existem estudos comparativos, randomizados, analisando os desfechos gestacionais de mulheres com história de colocação de Essure e IVF-ET posterior, em função do número de espirais deixadas visíveis na cavidade uterina após introdução do dispositivo. Os melhores trabalhos publicados $^{21-25}$ apresentam limitações importantes relacionadas com o poder amostral e desenhos para investigações multicêntricas serão, seguramente, necessários para esclarecer a segurança dos bons resultados obtidos até à data. Este aspecto reveste-se, na opinião dos autores, da maior importância. A particular preocupação com a ocorrência de

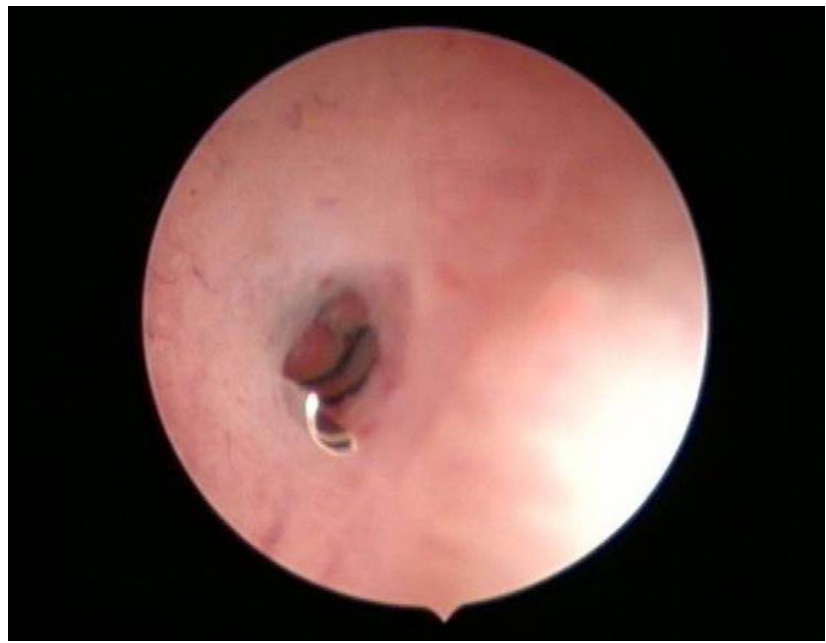

Figura 2 - Visualização do número de espirais visíveis na cavidade após colocação do dispositivo Essure ${ }^{\circledR}$ no ostium tubar da trompa afectada. No caso apresentado, apenas 1 espiral foi deixada visível na cavidade.

desfechos obstétricos desfavoráveis, relacionados com a existência de um corpo estranho na trompa abordada, ${ }^{21-25}$ justifica o objectivo de deixar um número mínimo de espirais na cavidade relevando, uma diminuição expectável dos riscos de potencial irritação endometrial e consequente menor receptividade (Fig. 2).

Efectivamente, o número de casos apresentados pelo estudo é insuficiente para recomendar a utilização prioritária da histeroscopia, com o objectivo de exclusão tubar, em vez da laparoscopia. De acordo com outros testemunhos presentes na literatura, ${ }^{21-25}$ doentes com contraindicação para anestesia geral, com risco acrescido de complicações cirúrgicas, particularmente pela maior incidência de patologia inflamatória pélvica crónica em mulheres com história de infertilidade expressa muitas vezes por aderências, ${ }^{34}$ a presença de múltiplas cirurgias abdominais anteriores e nos casos de obesidade grave (dificultando a realização de laparoscopia) são, na opinião dos autores, indicações potenciais para primeira escolha para oclusão tubar utilizando a via histeroscópica em vez da via laparoscópica.

Serão necessários mais estudos, nomeadamente comparando um grupo de doentes tratadas com Essure ${ }^{\circledR}$ com outro em que se proceda a oclusão da trompa afetada por via laparoscópica, para se avaliar a importância do método histeroscópico, mesmo nos casos sem contra-indicação para cirurgia laparoscópica.

No nosso centro, o protocolo para confirmação da posição normal dos implantes (Essure ${ }^{\circledR}$ ), consiste na realização de radiografia pélvica e ecografia bi/tridimensional, procurando na porção proximal da trompa ocluída a presença de um corpo estranho linear, hiperecogénico, cujo limite à cavidade uterina é facilmente objectivável. Pachy et al, ${ }^{35} \mathrm{em}$ 2009 , apresentou as vantagens da ecografia 3D com sonda vaginal no controle da posição dos dispositivos. A facilidade de realização do exame, a ausência de radiações e a sua reprodutibilidade, tem motivado alguns autores a adoptarem esta forma de controlo após colocação de Essure ${ }^{\circledR}$. Na 
verdade, até ao momento, não estão demonstrados benefícios concretos dos métodos radiológicos convencionais sobre a ecografia transvaginal (bi/tridimensional) na avaliação da normal posição dos implantes, ${ }^{36,37}$ embora muitos considerem a ausência de existência de um método seguro, fácil e reprodutível na avaliação da permeabilidade tubar quer após esterilização histeroscópica, quer após oclusão tubar uni/bilateral com o objectivo de realização de tratamentos de infertilidade.

\section{CONCLUSÃO}

À semelhança de outros estudos já realizados, ${ }^{21-25} \mathrm{com}$ séries muito semelhantes à apresentada neste trabalho, sugerimos a eficácia obtida pela oclusão tubar induzida pelo dispositivo Essure ${ }^{\circledR}$, de forma unilateral, nos resultados do tratamento de mulheres com história de infertilidade associada a hidrossalpinges unilaterais, visíveis no exame ecográfico transvaginal, submetidas a IVF-ET. O número reduzido de doentes que compõem a amostra dos estudos até então realizados, justifica a necessidade de estudos multicêntricos. Particularmente, faltam estudos randomizados esclarecendo se existem benefícios da oclusão tubar por via laparoscópica versus via histeroscópia (colocação de Essure ${ }^{\circledR}$ ). Particular relevância deverá ser dada à análise da segurança da liga metálica do dispositivo Essure ${ }^{\circledR}$ sobre os desfechos gestacionais/perinatais.

Na série apresentada pelo nosso estudo, a ocorrência de um caso de gravidez espontânea em mulher com oito anos de infertilidade, após colocação do dispositivo de forma unilateral, favorece de forma objectiva, a teoria de que o fluido tubar prejudica a ocorrência de gravidez, aspecto já bem documentado em hidrossalpinges visíveis ecograficamente ${ }^{3,5,8,13,19,21,24}$. Não foram identificados efeitos negativos da presença de Essure ${ }^{\circledR}$ sobre a progressão da gravidez em qualquer um dos casos apresentados.

\section{CONFLITOS DE INTERESSE}

Os autores não declararam quaisquer conflitos de interesse relativamente ao presente artigo.

\section{FONTES DE FINANCIAMENTO}

Os autores não mencionaram quaisquer fontes externas de financiamento.

\section{REFERÊNCIAS}

1. loechle M. What is a hydrosalpinx? A plea for the use of a proper terminology in scientific discussion. Hum Reprod. 1999;14:578.

2. Strandell A. The influence of hydrosalpinx on IVF and embryo transfer: a review. Hum Reprod Update. 2000;6:387-95.

3. He RH, Gao HJ, Li YQ, Zhu XM. The associated factors to endometrial cavity fluid and the relevant impact on the IVF-ET outcome. Reprod Biol Endocrinol. 2010;8:46.

4. Sharara FI. Ultrasound-guided hydrosalpinx aspiration during oocyte collection improves outcome in IVF. Hum Reprod. 2009;24:756.

5. Hammadieh N, Coomarasamy A, Ola B, Papaioannou S, Afnan M, Sharif $\mathrm{K}$. Ultrasound-guided hydrosalpinx aspiration during oocyte collection improves pregnancy outcome in IVF: a randomized controlled trial. Hum Reprod. 2008;23:1113-7.

6. Hammadieh N, Afnan M, Evans J, Sharif K, Amso N, Olufowobi O. A postal survey of hydrosalpinx management prior to IVF in the United Kingdom. Hum Reprod. 2004;19:1009-12.

7. Ajonuma LC, $\mathrm{Ng} \mathrm{EH}$, Chan $\mathrm{HC}$. New insights into the mechanisms underlying hydrosalpinx fluid formation and its adverse effect on IVF outcome. Hum Reprod Update. 2002;8:255-64.

8. Strandell A, Lindhard A. Why does hydrosalpinx reduce fertility? The importance of hydrosalpinx fluid. Hum Reprod. 2002;17:1141-5.

9. $\mathrm{Ng} \mathrm{EH}$, Ajonuma LC, Lau EY, Yeung WS, Ho PC. Adverse effects of hydrosalpinx fluid on sperm motility and survival. Hum Reprod. 2000;15:772-7

10. Edwards RG, Steptoe PC, Purdy JM. Establishing full term human pregnancies using cleaving embryos grown in vitro. $\mathrm{Br} \mathrm{J}$ Obstet Gynecol. 1980;87:737-56.

11. Barmat LI, Rauch E, Spandorfer S, Kowalik A, Sills ES, Schattman G, et al. The effect of hydrosalpinges on IVF-ET outcome. J Assist Reprod Genet. 1999;16:350-4.

12. Sagoskin AW, Lessey BA, Mottla GL, Richter KS, Chetkowski RJ, Chang AS, et al. Salpingectomy or proximal tubal occlusion of unilateral hydrosalpinx increases the potential for spontaneous pregnancy. Hum Reprod. 2003;18:2634-7.

13. Aboulghar MA, Mansour RT, Serour GI. Spontaneous intrauterine pregnancy following salpingectomy for a unilateral hydrosalpinx. Hum Reprod. 2002;17:1099-100.

14. Johnson NP, Mak W, Sowter MC. Laparoscopic salpingectomy for women with hydrosalpinges enhances the success of IVF: a Cochrane review. Hum Reprod. 2002;17:543-8.

15. Strandell A, Lindhard A, Waldenström U, Thorburn J. Hydrosalpinx and IVF outcome: cumulative results after salpingectomy in a randomized controlled trial. Hum Reprod. 2001;16:2403-10.
16. Strandell A, Lindhard A, Waldenström U, Thorburn J. Prophylactic salpingectomy does not impair the ovarian response in IVF treatment. Hum Reprod. 2001;16:1135-9.

17. Strandell A, Lindhard A. Hydrosalpinx and ART. Salpingectomy prior to IVF can be recommended to a well-defined subgroup of patients. Hum Reprod. 2000;15:2072-4.

18. Puttemans P, Campo R, Gordts S, Brosens I. Hydrosalpinx and ART: hydrosalpinx--functional surgery or salpingectomy? Hum Reprod. 2000;15:1427-30.

19. Strandell A, Lindhard A, Waldenström U, Thorburn J, Janson PO, Hamberger L. Hydrosalpinx and IVF outcome: a prospective, randomized multicentre trial in Scandinavia on salpingectomy prior to IVF. Hum Reprod. 1999;14:2762-9.

20. Na ED, Cha DH, Cho JH, Kim MK. Comparison of IVF-ET outcomes in patients with hydrosalpinx pretreated with either sclerotherapy or laparoscopic salpingectomy. Clin Exp Reprod Med. 2012;39:182-6.

21. Sonigo $C$, Collinet $P$, Rubod $C$, Catteau-Jonard S. Place actuelle des micro-implants Essure ${ }^{\circledR}$ dans la prise en charge des hydrosalpinx avant fécondation in vitro. Gynecol Obstet Fertil. 2013;41:133-8.

22. Thébault N, Broux PL, Moy L, Vialard J. Utilization of Essure ${ }^{\circledR}$ microinsert for hydrosalpynx occlusion in infertile women. J Gynecol Obstet Biol Reprod. 2012;41:145-50

23. Mijatovic V, Dreyer K, Emanuel MH, Schats R, Hompes PG. Essure ${ }^{\circledR}$ Hydrosalpinx occlusion prior to IVF-ET as an alternative to laparoscopic salpingectomy. Eur J Obstet Gynecol Reprod Biol. 2012;161:42-5.

24. Mijatovic V, Veersema S, Emanuel MH, Schats R, Hompes PG. Essure Hysteroscopic tubal occlusion device for the treatment of hydrosalpinx prior to in vitro fertilization-embryo transfer in patients with a contraindication for laparoscopy. Fertil Steril. 2010;93:1338-42.

25. Hitkari JA, Singh SS, Shapiro HM, Leyland N. Essure treatment of hydrosalpinges. Fertil Steril. 2007;88:1663-6.

26. Aboulghar MA, Mansour RT, Serour GI. Controversies in the modern management of hydrosalpinx. Hum Reprod Update. 1998;4:882-90.

27. Johnson N, van Voorst S, Sowter MC, Strandell A, Mol BW. Surgical treatment for tubal disease in women due to undergo in vitro fertilisation. Cochrane Database Syst Rev. 2010;20.

28. Parihar M, Mirge A, Hasabe R. Hydrosalpinx functional surgery or salpingectomy? The importance of hydrosalpinx fluid in assisted reproductive technologies. J Gynecol Endosc Surg. 2009;1:12-6.

29. Eytan O, Azem F, Gull I, Wolman I, Elad D, Jaffa AJ. The mechanism of hydrosalpinx in embryo implantation. Hum Reprod. 2001;16:2662-7.

30. Kraemer DF, Yen PY, Nichols M. An economic comparison of female sterilization of hysteroscopic tubal occlusion with laparoscopic bilateral 
tubal ligation. Contraception. 2009;80:254-60.

31. Hopkins MR, Creedon DJ, Wagie AE, Williams AR, Famuyide AO. Retrospective cost analysis comparing Essure hysteroscopic sterilization and laparoscopic bilateral tubal coagulation. J Minim Invasive Gynecol. 2007; 14:97-102.

32. Levie MD, Chudnoff SG. Office hysteroscopic sterilization compared with laparoscopic sterilization: a critical cost analysis. J Minim Invasive Gynecol. 2005;12:318-22

33. Wever DJ, Veldhuizen AG, Sanders MM, Schakenraad JM, van Horn JR. Cytotoxic,allergic and genotoxic activity of a nickel-titanium alloy. Biomaterials. 1997;18:1115-20.
34. Torre A, Pouly JL, Wainer B. Anatomic evaluation of the female of the infertile couple. J Gynecol Obstet Biol Reprod. 2010;39:34-44.

35. Pachy F, Bardou D, Piovesan P, Jeny R. Vaginal tridimensionel ultrasound interest for the assessment of correct Essure sterilization microinsert placement. J Gynecol Obstet Biol Reprod. 2009;38:321-7.

36. Kerin JF, Levy BS. Ultrasound: an effective method for localization of the echogenic Essure sterilization micro-insert: correlation with radiologic evaluations. J Minim Invasive Gynecol. 2005;12:50-4.

37. Veersema S, Vleugels M, Koks C, Thurkow A, van der Vaart H, Brölmann $\mathrm{H}$. Confirmation of Essure placement using transvaginal ultrasound. J Minim Invasive Gynecol. 2011;18:164-8. 


\section{Gravidezes Bem Sucedidas com Recurso a Fertilização In Vitro após Oclusão de Hidrossalpinge Unilateral com Essure ${ }^{\circledR}$ Acta Med Port 2014:27:342-348}

Publicado pela Acta Médica Portuguesa, a Revista Científica da Ordem dos Médicos

Av. Almirante Gago Coutinho, 151

1749-084 Lisboa, Portugal.

Tel: +351218428 215

E-mail: submissao@actamedicaportuguesa.com

www.actamedicaportuguesa.com

ISSN:0870-399X | e-ISSN: 1646-0758

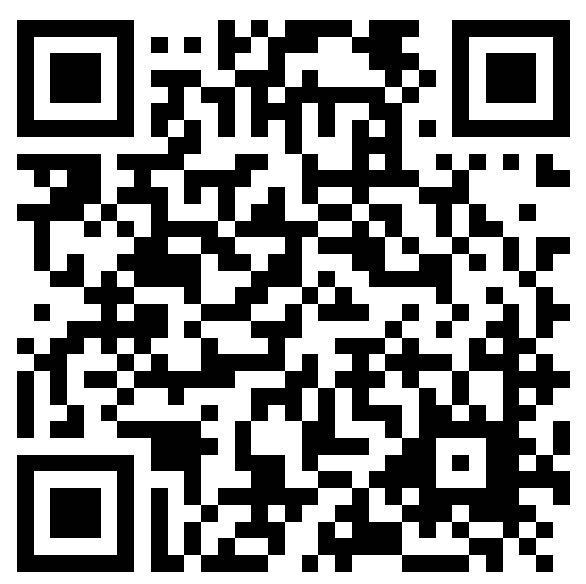

